\title{
Takotsubo syndrome versus myocardial infarction: what is the role of echocardiography?
}

\author{
Raquel Menezes Fernandes ${ }^{1,2 *}$, Hugo Costa ${ }^{1,2}$, Teresa Mota ${ }^{1,2}$, João Bispo ${ }^{1,2}$, Pedro Azevedo ${ }^{1,2}$, Dina Bento ${ }^{1,2,3}$, Nuno Marques ${ }^{1,2,3}$ \\ ${ }^{1}$ Cardiology Department, Centro Hospitalar Universitário do Algarve - Faro, Portugal \\ ${ }^{2}$ Algarve Biomedical Center, Faro, Portugal \\ ${ }^{3}$ Biomedical Sciences and Medicine Department, University of Algarve
}

${ }^{\star}$ Corresponding author: Raquel Menezes Fernandes, Cardiology Department, Centro Hospitalar Universitário do Algarve - Faro,

Portugal. Email: ana.raquel.mf@gmail.com

Received date: August 03, 2019; Accepted date: August 29, 2019; Published date: October 18, 2019

Citation: Raquel MF., Costa H., Mota T., Bispo J., Azevedo P. et al. (2019) Takotsubo Syndrome Versus Myocardial Infarction: what is the role of Echocardiography?, Clinical Cardiology and Cardiovascular Interventions, 2(2); DOI: 10.31579/ 2641-0419/017

Copyright: () 2019 Raquel M Fernandes, This is an open-access article distributed under the terms of the Creative Commons Attribution

License, which permits unrestricted use, distribution, and reproduction in any medium, provided the original author and source are credited.

\section{Abstract}

Reversible left ventricle dysfunction is a key feature of Takotsubo syndrome (TTS), but in the acute phase it can mimic an acute coronary syndrome, making this differentiation a significant challenge. Echocardiography is widely used in the early evaluation of these patients, and advanced imaging tools have highlighted the differences between both syndromes. The symmetrical pattern of left ventricular dysfunction, extending beyond the territory of a single coronary artery, associated with a less severe diastolic dysfunction, right ventricle involvement and a typical complete recovery of wall-motion abnormalities points towards the diagnosis of TTS. Despite all the imaging advances made so far, evaluation of coronary anatomy remains mandatory in these patients.

Keywords: takotsubo syndrome; stress-induced cardiomyopathy; acute myocardial infarction; echocardiography; speckle tracking; global longitudinal strain

\section{Abbreviations:}

2D STE $=$ Two-dimensional speckle tracking echocardiography

ACS $=$ Acute coronary syndrome AMI = Acute myocardial infarction

$\mathrm{CFR}=$ Coronary flow reserve

$\mathrm{LAD}=$ Left anterior descending $\mathrm{LV}=$ Left ventricle

$\mathrm{LVEF}=$ Left ventricle ejection fraction

LVOTO $=$ Left ventricle outflow tract obstruction NSTEMI $=$ Non-ST elevation myocardial infarction PSS = Post- systolic shortening; RV = Right ventricle SAM = Systolic anterior motion

TAPSE $=$ Tricuspid annular plane systolic excursion TTS $=$ Takotsubo syndrome

WMA $=$ Wall motion abnormalities

\section{Introduction}

Takotsubo syndrome (TTS), also known as stress-induced cardiomyopathy, is an acute syndrome characterized by transient left ventricle (LV) dysfunction, in the absence of obstructive coronary artery disease [2,3]. It typically occurs in postmenopausal women and is often triggered by an emotional or physical stressful event. TTS may account for $1 \%-2 \%$ of all patients admitted with a presumed diagnosis of acute coronary syndrome (ACS) [2], but this rate increases to $6-7 \%$ if only females are considered [3].

Differentiating TTS from acute myocardial infarction (AMI) before coronariography proves to be a real challenge, since both share similar presentation, electrocardiographic, and laboratory abnormalities [2,4,5]. TTS is a diagnosis of exclusion, so most patients are initially diagnosed as having an ACS, but do not have significant coronary artery lesions to explain the segmental abnormalities [6]. The greatest difficulty may be in distinguishing apical TTS (classical form) from non-ST-elevation myocardial infarction (NTSEMI) with left anterior descending (LAD) coronary artery disease $[7,8]$, in which a coronariography is not emergent

\section{Conventional echocardiography}

Due to its widespread availability, echocardiography is frequently the first non-invasive imaging modality used for early evaluation of LV systolic function in TTS patients [9]. It exhibits strong correlation with left ventriculography and detects potential complications such as mitral valve regurgitation, outflow tract obstruction, thrombus, LV free wall rupture and right ventricle (RV) involvement [9-12]. The main difficulty is the differentiation between classic TTS and anterior AMI, due to somewhat overlapping echocardiographic abnormalities.

LV dysfunction is a key feature in both TTS and AMI patients, but TTS is associated with worse LV systolic dysfunction and diffuse regional wall- 
motion abnormalities (WMA) at the acute phase, despite only a slight increase in troponin values[3,5,8]. TTS reveals a distinctive pattern of contractility characterized by symmetrical and circumferential WMA, irrespective of the epicardial vascular territory distribution (noncoronary pattern), suggestive of extensive myocardial stunning [2-4,9-13] . On the contrary, AMI primarily affects the territories supplied by the "culprit" coronary artery [9]. According to Citro el al, a wall motion score index (WMSI) of $\geq 1.75$ has a high positive predictive value for the diagnosis of TTS, reinforcing the concept of diffuse injury [3]. WMSI values are higher in TTS patients with ST-segment elevation, indicating more severe myocardial dysfunction [3].

Classical TTS presents with mid- ventricular WMA (involving anterior, inferior, and lateral walls), apical akinesia, preserving or increasing the contractile function of the basal LV segments [9,14] (Figure 1). Midcavity, basal segmental (reverse takotsubo), focal, biventricular and isolated RV variants have also been described, being less frequent $[8,10,15]$. However, estimation of the LV ejection fraction (LVEF) using the modified Simpson's rule does not yield the complexity of LV mechanics, since it only assesses the contractility in its radial direction $[1,16]$.

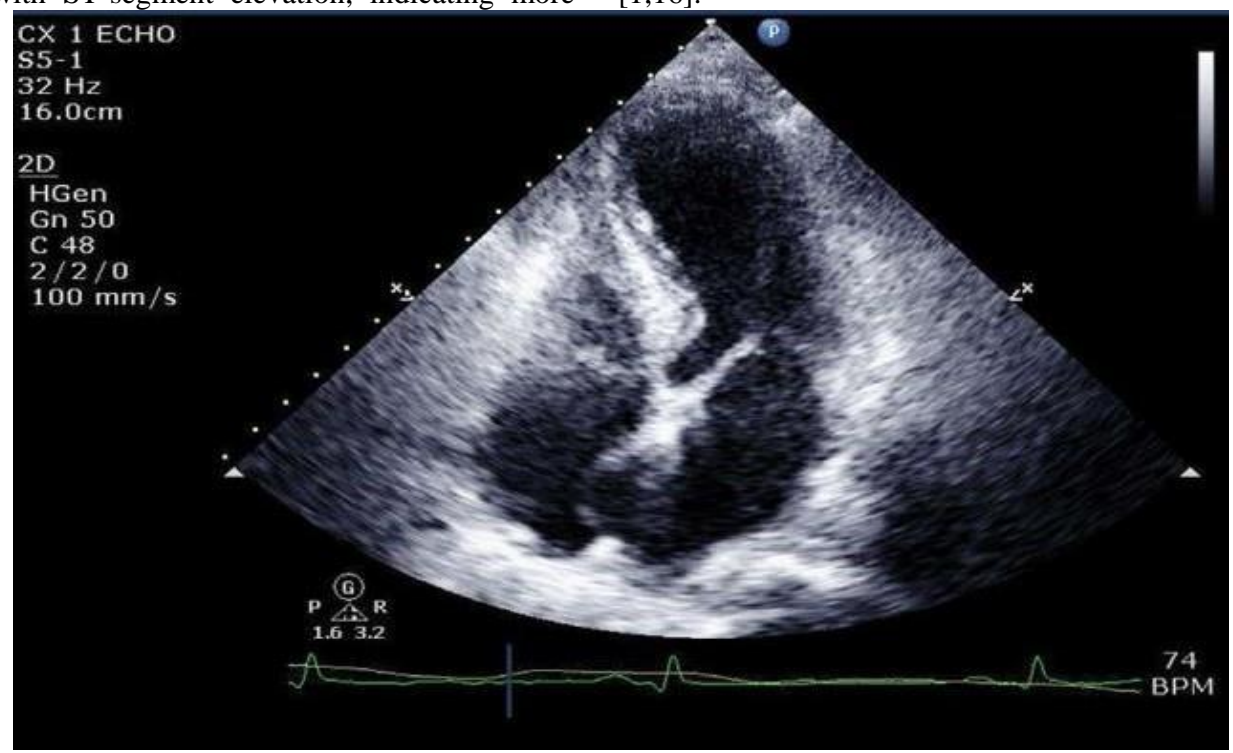

Figure 1: Transthoracic echocardiography in Takotsubo syndrome - classic form, viewed in end-systole at 4- chamber apical incidence (Weiner et al, 2017).

\section{Speckle Tracking Strain Echocardiography}

Two-dimensional speckle tracking echocardiography (2D STE) is a tool for quantitative assessment of myocardial function [13,16-18]. Through measurement of myocardial strain, it has better reproducibility than tissue Doppler imaging strain and higher sensitivity in detecting subtle myocardial abnormalities [1,9], revealing myocardial dysfunction even before significant changes in LVEF and cardiac output [19]. Longitudinal strain has proven to be the most sensitive and reproducible of the various strain measurements performed with speckle tracking, and can provide important prognostic information $[10,19]$.

In TTS patients, similar velocities are recorded among all basal LV segments, middle segments, and apical segments, translating in a circular LV dysfunction. In patients with anterior AMI, there are significant differences between those segments, affecting specifically the LAD coronary artery perfusion territory. These findings suggest that TTS is not the consequence of transient LAD occlusion [13].

In parallel, Heggemann et al showed uniform reduction of radial strain along the entire mid LV circumference in TTS, comparing with significantly higher values in AMI posterior, lateral and inferior segments [4]. Similarly, longitudinal strain in some of the posterior, inferior and lateral segments is significantly lower in TTS compared to anterior AMI, adding to a greater involvement of the median and apical segments, resulting in a lower global longitudinal strain [4] (Figure 2).

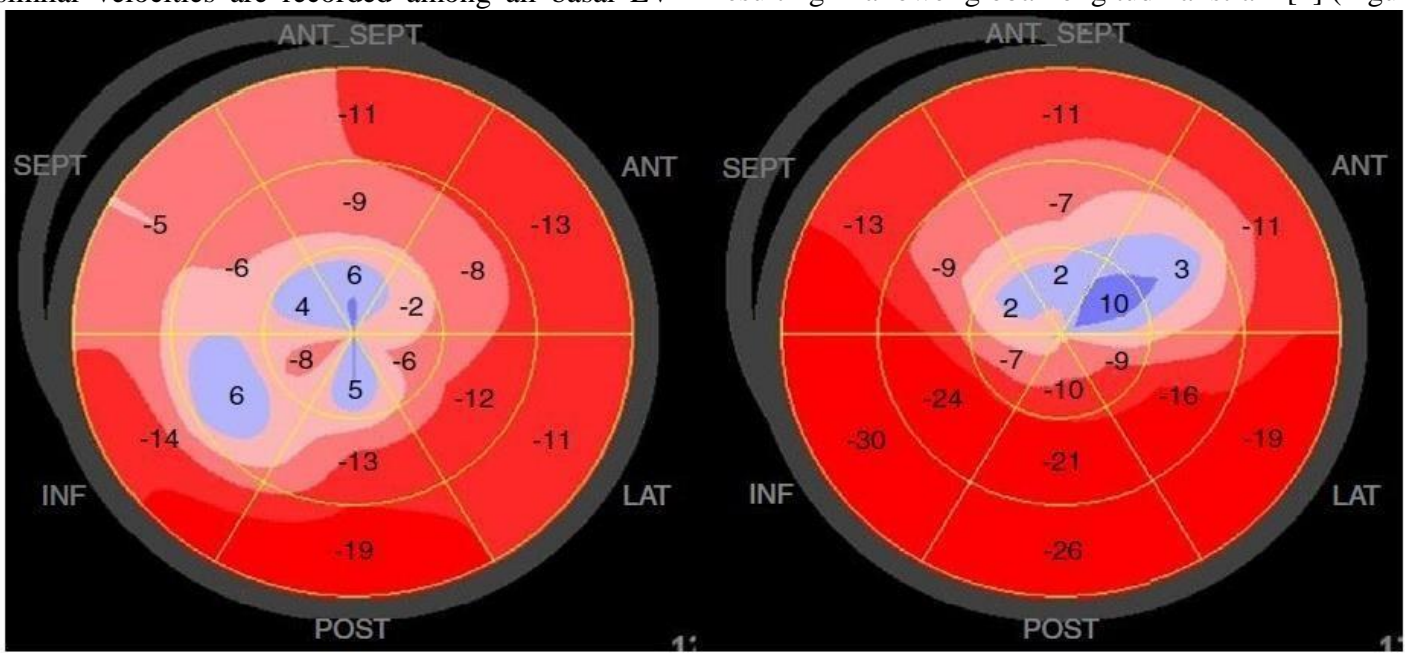

Figure 2: Polar maps of peak systolic longitudinal strain obtained from a Takotsubo syndrome patient (left) and an anterior ST-segment elevation myocardial infarction patient (right) (Pestana et al, 2019). 
LV twist parameters are preserved in basal segments, while apical twist during systole and diastole are significantly reduced in TTS, compatible with a transmural impairment of LV function [1,5]. Longitudinal strain of the LV base could also be diminished in several segments, notwithstanding the basal hypercontractility perception in TTS at baseline [20]. Despite previous reports of a significant apex-to-base gradient of strain [20], Pestana et al found a smaller basal/apical longitudinal strain ratio in TTS patients, indicative of a more generalized myocardial dysfunction [8].

Post-systolic shortening (PSS) is defined as a further shortening / thickening occurring after the end-systole. It is a non-specific, but sensitive, marker of regional myocardial dysfunction and reflects a subtle systolic stunning that cannot be detected by conventional systolic Parameters $[14,20]$. Pathological PSS can be found in $78 \%$ of acutely ischaemic myocardial segments, with a similar incidence during the acute phase of TTS [20].

Three-dimensional (3D) speckle tracking echocardiography is feasible to assess regional LV function and its recovery in TTS. Consistent with WMA in TTS, Kobayashi et al found that the average peak systolic 3D radial strain value of LV base is higher than those of mid and apex at baseline, decreasing significantly after six months, whereas those of mid and apex increase significantly [14].

\section{Coronary microcirculation assessment}

Non-invasive evaluation of distal LAD flow by doppler echocardiography could also help differentiate TTS from AMI [21]. LAD flow is expected to be absent in anterior AMI due to artery occlusion, but is detectable in TTS [9] (Figure 3). Diastolic to systolic velocity ratio of the LAD flow is decreased in patients with significant LAD stenosis, and may also be useful in this differentiation [22].

In addition, coronary flow reserve (CFR) is transiently impaired in TTS [22], but at a lower grade compared with AMI, despite a poorer WMSI, suggesting the involvement of other mechanisms than direct microcirculatory damage in the pathogenesis of WMA [21]. At follow-up, there is an improvement of nearly $40 \%$ of CFR (above of the $10 \%$ of intraindividual variability), correlating with the recovery of LV systolic function [9]. Current evidence does not allow the reliable distinction of the two entities through this method, translating in a limited utility of the non-invasive evaluation of coronary assessment at the present day.

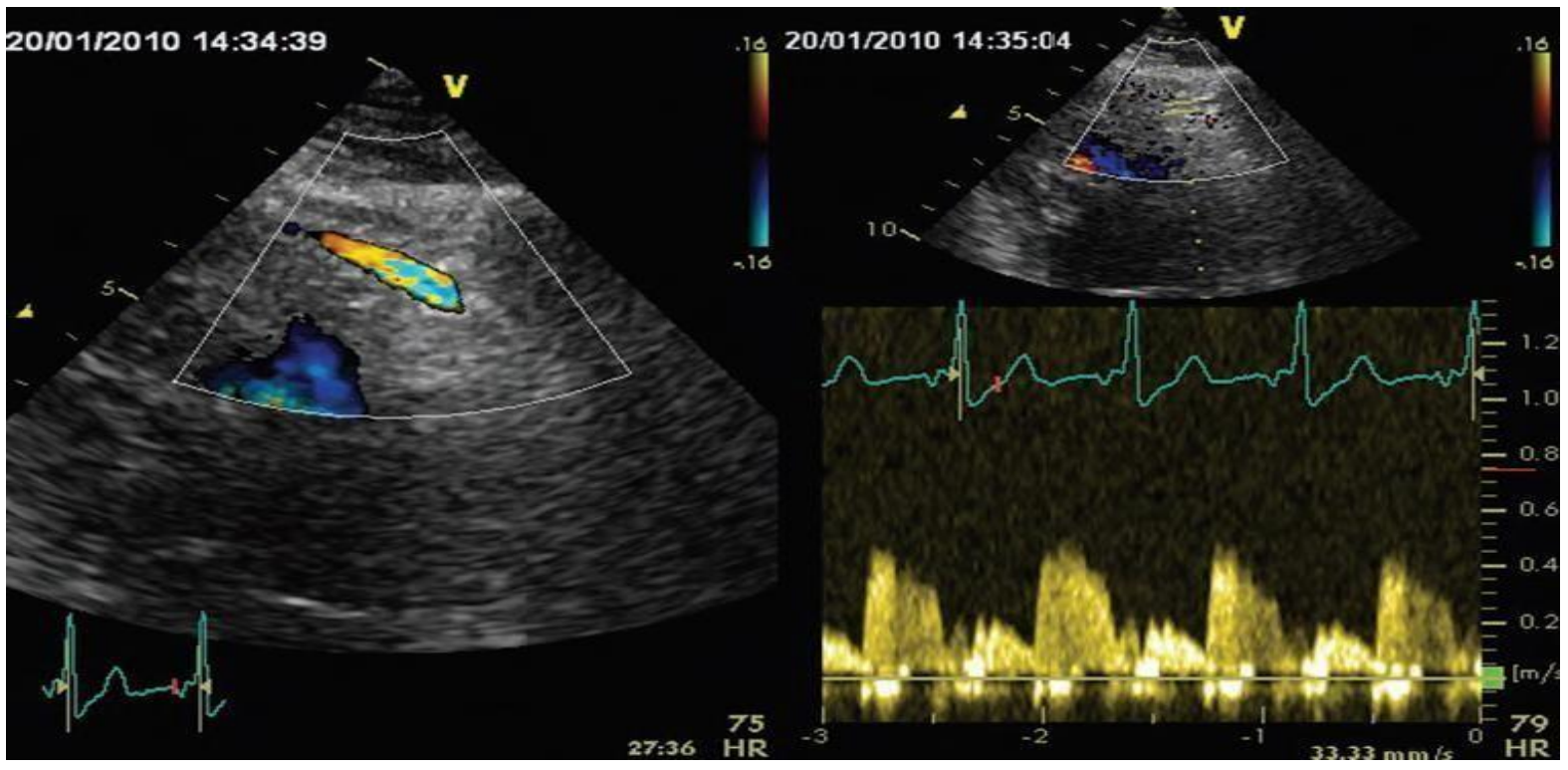

Figure 3: Visualization of the distal LAD flow by colour Doppler and the corresponding coronary flow velocity recorded with the pulsed- wave Doppler sampling (Meimoun et al, 2013).

\section{Diastolic function}

Global and regional diastolic dysfunction has been observed in the early phase of TTS, as evidenced by impaired LV untwisting and increased E/e' ratio [9]. Despite the worse systolic function in TTS patients, LV filling pressure appears to be lower than in those with AMI, which explains better Clinical outcome in the former patients [23]. Increased stiffness of akinetic segments is possibly related to scar formation or more transmural involvement in AMI. Diastolic function parameters appear to have a good correlation with the extent of myocardial fibrosis and viability [23].

\section{LV outflow obstruction and mitral regurgitation}

In some patients with TTS, systolic anterior motion (SAM) and LV outflow tract obstruction (LVOTO) may develop as a result of LV basal wall hyperkinesis, occurring in about $10 \%$ to $25 \%$ [11,17,22] (Figure 4). It typically resolves with medical management and gradual ventricular recovery [11,22]. Mitral regurgitation may also develop as a result of two different mechanisms: tethering and SAM of the anterior mitral leaflet. Transient LV dilatation with or without mitral annular dilatation may cause apical tethering of the mitral leaflets, resulting in decrease leaflet coaptation and a Carpentier III mechanism [11,22]. 


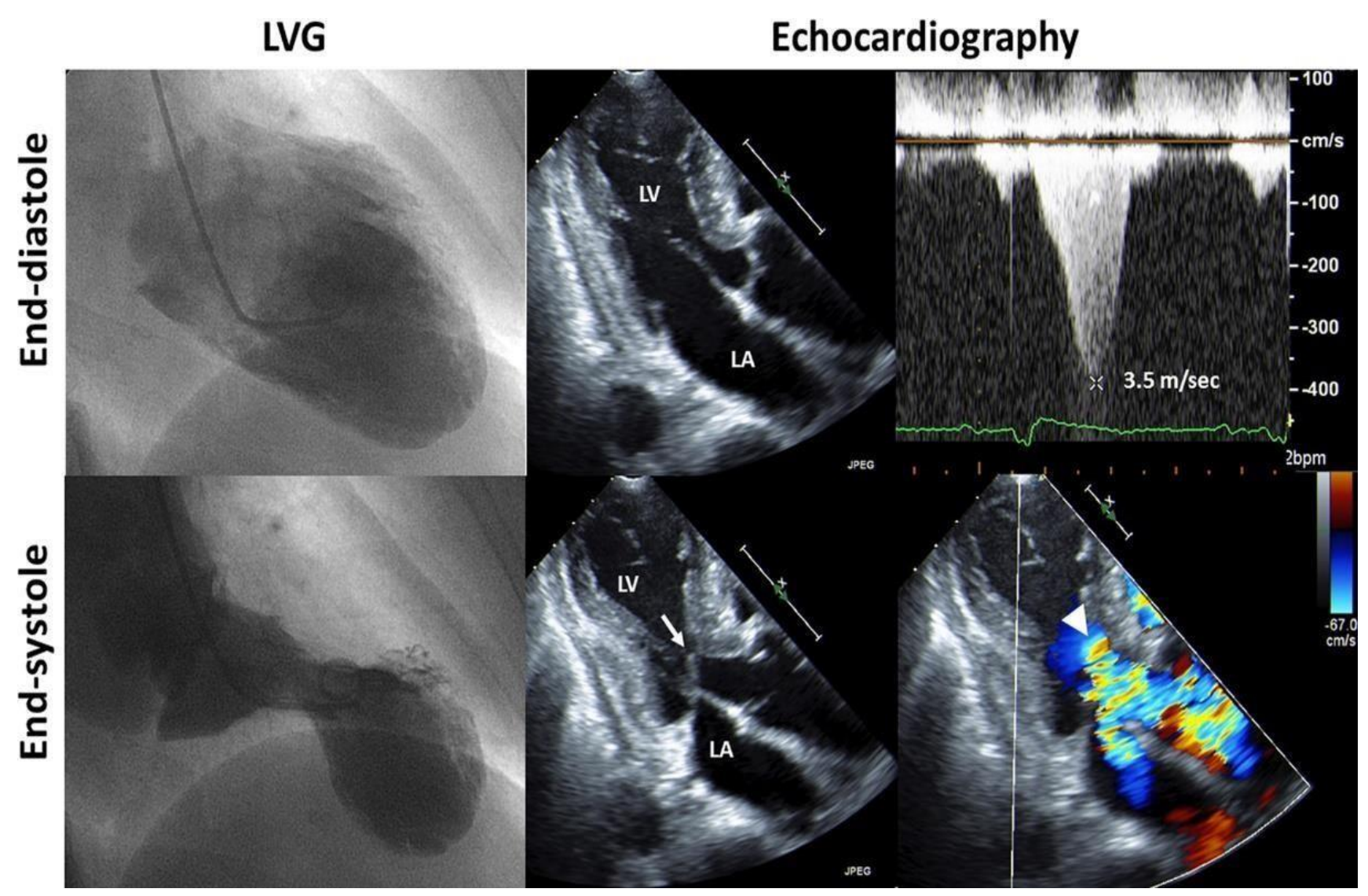

Figure 4: Left ventriculography (LVG) and echocardiography in TTS patient with SAM, mitral regurgitation and LVOTO (Okura et al, 2015).

\section{Right ventricle involvement}

A careful assessment of global and regional RV function should be recommended, since about one-quarter of patients with TTS shows RV impairment, uncommon in LAD disease [2,3,11,22]. The pattern of RV regional WMA is similar to the $\mathrm{LV}$, so a right ventricular or biventricular "ballooning" may be observed [3,24]. Impaired global RV function in TTS seems to be predominantly caused by mid-ventricular and apical RV free wall strain reduction [24].

Strain imaging has higher sensitivity in the quantification of RV function than the global parameters RV fractional area change and tricuspid annular plane systolic excursion (TAPSE) [24]. TAPSE and RVS' may not be suitable to perceive isolated abnormalities in distal RV segments. Global longitudinal strain detects RV involvement in TTS at a rate of $58 \%$, the highest among all the parameters [2] (Figure 5). The group of Heggemann et al found that RV PSS $>-19.1 \%$ indicates RV dysfunction in TTS patients, with a sensitivity of $85 \%$ and specificity of $71 \%$. However, it only accounts the RV free wall [24].RV involvement in TTS is associated with significantly lower LVEF and lower LV global strain at baseline, being also an important prognostic factor, as these patients are generally older and have a more severe clinical course [24,25]. 

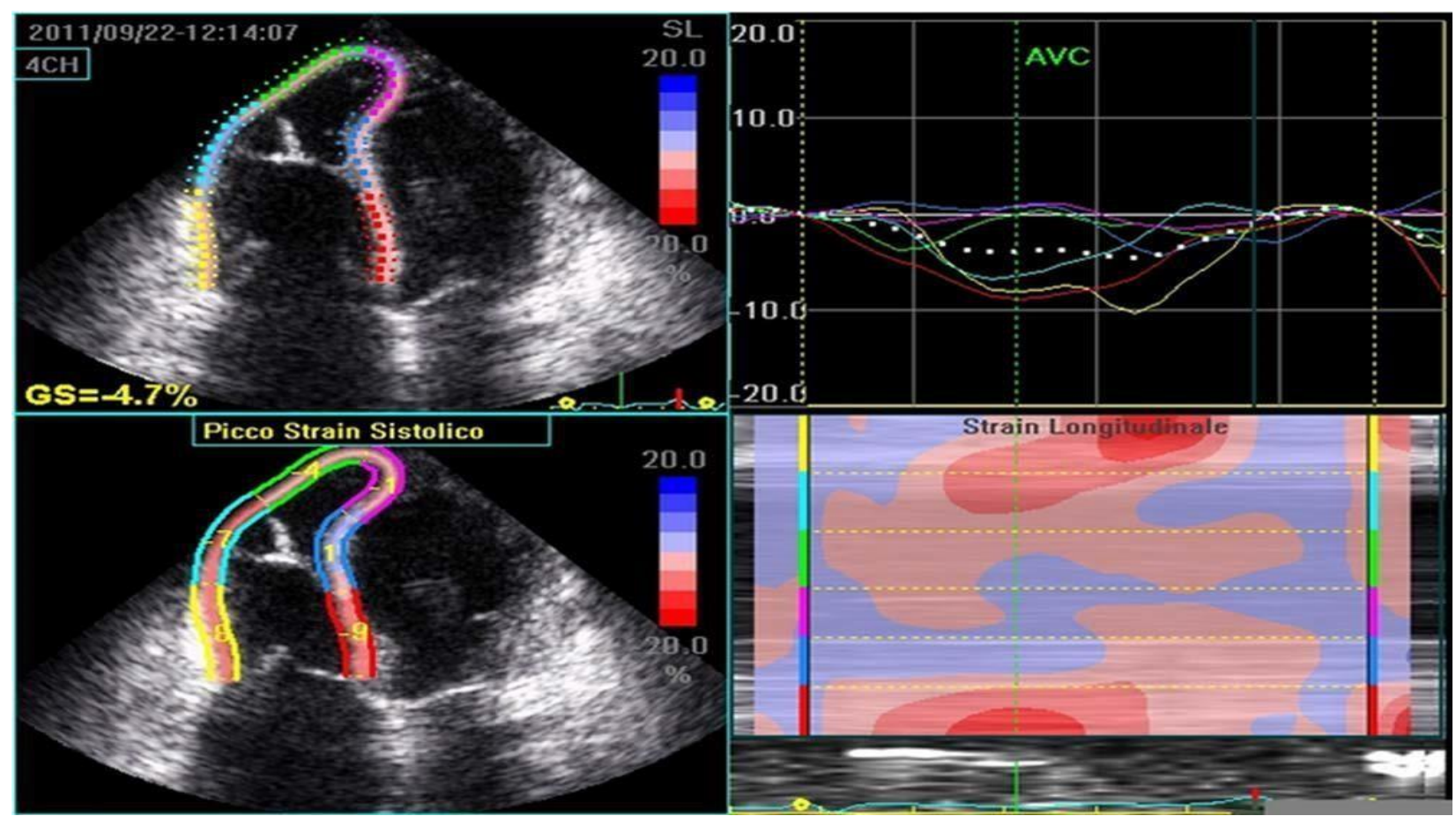

Figure 5. Two-dimensional longitudinal strain curve analysis of the RV and corresponding parametric image (two-dimensional and color M-mode) in a patient with biventricular TTS (Vizzardi et al, 2014).

\section{Stress echocardiography}

Citro et al refer to a potential role of low-dose dobutamine stress echocardiography in assessing reversibility of myocardial dysfunction in suspected TTS, through demonstration of myocardial viability [9]. However, because of the role of catecholamines in inducing TTS, they also state that dobutamine should be administered with caution and probably avoided in the early phase $[9,16]$. It also has the potential to exacerbate or induce LVOTO, in particular in patients with myocardial hypertrophy, leading to an increased risk for hemodynamic deterioration and associated complications [9]. Current evidence doesn't support the routine use of stress echocardiography in the diagnostic management of TTS patients.

\section{Recovery}

Despite the rapid resolution of WMA and LVEF [5,14] subtle LV dysfunction seem to persist for a few weeks, indicating that complete recovery may not occur as rapidly as previously though [20,27]. 2D strain can still be lower comparing to controls, emphasizing that LVEF is insufficient in quantifying intrinsic myocardial contractility [27] (Figure 6). Furthermore, Heggemann et al state that more than half of LV segments also showed PSS even with WMA normalization, suggesting that PSS could be an indicator of persisting abnormalities of LV regional function into the early recovery period $[2$

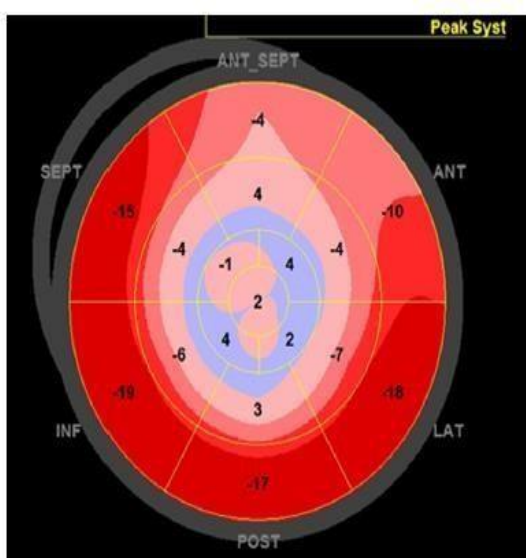

GLPSS $-8.9 \%$

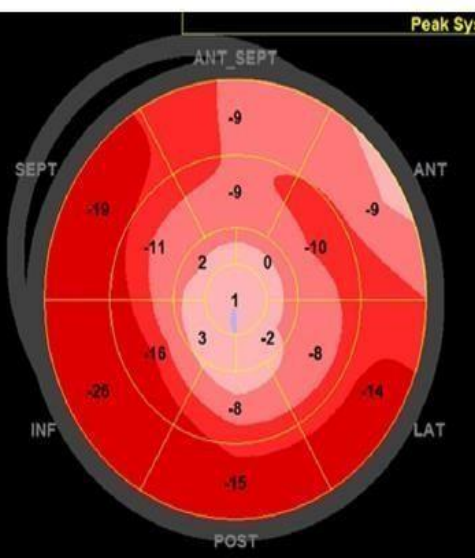

GLPSS $-10.6 \%$

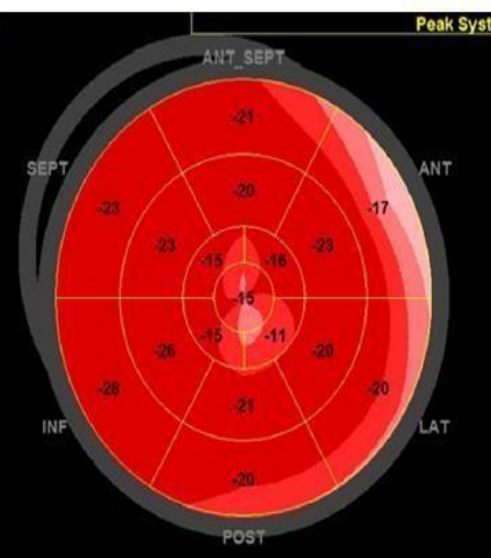

GLPSS - $18.1 \%$

Day 1

Day 4

Day 21

Figure 6. An example of bull's eye plot of peak systolic strain in a patient with TTS, at admission and during follow-up. GLPSS = global longitudinal peak systolic strain (Kim et al, 2016). 
Even though TTS patients present circular LV dysfunction, the recovery pattern is not uniform [13,21]. For example, in mid-LV, longitudinal strain showed slower recovery than circumferential strain [25]. Due to this asymmetrical and partial recovery of segmental contractility a few days later, TTS may mimic LV dysfunction observed in patients with coronary artery disease, making the distinction from AMI more challenging if a patient is evaluated only at this stage $[9,13]$. Temporal evolution of TTS is variable, remaining to be determined the exact time-course of the recovery process (possibly 21 days to 6 months) and whether these findings have potential therapeutic and prognostic implications $[1,20]$. Table 1 summarizes the main differences between TTS and AMI discussed in this review.

\begin{tabular}{|c|c|c|}
\hline & Takotsubo syndrome (classic form) & (anterior) Acute myocardial infarction \\
\hline Ejection fraction & Significantly reduced & Reduced \\
\hline $\begin{array}{l}\text { Wall motion- } \\
\text { abnormalities }\end{array}$ & $\begin{array}{l}\text { Symmetrical, circumferential; apical } \\
\text { ballooning with basal hyperkinesis in } \\
\text { classic form }\end{array}$ & $\begin{array}{c}\text { Follows a coronary artery perfusion } \\
\text { territory (anterior wall and apex in } \\
\text { anterior AMI) }\end{array}$ \\
\hline $\begin{array}{l}\text { Wall motion score } \\
\text { index }\end{array}$ & Significantly elevated & Elevated \\
\hline $\begin{array}{l}\text { LV outflow tract } \\
\text { obstruction }\end{array}$ & $\begin{array}{l}\text { Could be present and associated with } \\
\text { SAM of the anterior mitral leaflet }\end{array}$ & Absent \\
\hline $\begin{array}{c}\text { Mitral } \\
\text { regurgitation }\end{array}$ & Due to SAM or tethering & $\begin{array}{l}\text { Due to tethering of mitral leaflets, } \\
\text { with or without annular dilation }\end{array}$ \\
\hline $\begin{array}{c}\text { Diastolic } \\
\text { dysfunction }\end{array}$ & Not as severe as systolic dysfunction & Significant \\
\hline $\begin{array}{l}\text { Right ventricle (RV) } \\
\text { involvement }\end{array}$ & $\begin{array}{l}\text { Reaches } 25 \% \text {; RV "ballooning"; Reduced } \\
\text { global longitudinal strain, with reduced } \\
\text { strain in apical segments; }\end{array}$ & Uncommon \\
\hline Radial strain & $\begin{array}{l}\text { Reduced in all mid-ventricular segments } \\
\text { (classic form) }\end{array}$ & $\begin{array}{c}\text { Reduced in the anterior and } \\
\text { anteroseptal wall (anterior AMI) }\end{array}$ \\
\hline $\begin{array}{c}\text { Global } \\
\text { longitudinal strain }\end{array}$ & Significantly lower at admission & Lower \\
\hline LAD flow & Present & Absent if LAD occlusion \\
\hline $\begin{array}{l}\text { Coronary flow } \\
\text { reserve }\end{array}$ & Transiently impaired, but at a lower grade & Severely impaired \\
\hline Recovery & $\begin{array}{l}\text { Complete; Asymmetrical; Subtle strain } \\
\text { alterations could persist for a few months }\end{array}$ & $\begin{array}{l}\text { Not as rapidly and usually not } \\
\text { complete }\end{array}$ \\
\hline
\end{tabular}

Table 1. Summary of echocardiographic differences between Takotsubo syndrome and acute myocardial infarction

$\mathrm{LAD}=$ left anterior descending coronary artery; $\mathrm{LV}=$ Left ventricle; $\mathrm{SAM}=$ Systolic anterior motion; $\mathrm{RV}=\mathrm{Right}$ ventricle

\section{Conclusions}

Patients that present with a suggestive clinical picture and an acute STsegment elevation on the electrocardiogram require emergent coronary angiogram. On the other hand, in the absence of ST-segment elevation and if clinically appropriate, the differential diagnosis between AMI and TTS could be made, to obviate unnecessary invasive procedures. Although the echocardiographic findings of TTS could mimic those of AMI, the regional WMA pattern, myocardial strain, diastolic dysfunction and degree of RV involvement differ. LV dysfunction is completely reversible in TTS, even though the recovery duration seems to be variable. Currently, despite the presence of echocardiographic findings suggestive of TTS, coronary angiography remains essential for the definitive diagnosis. Alternatively, and if available, cardiac computed tomographic angiography could be applied to exclude high-grade coronary artery stenosis in patients with a highly suggestive clinical picture of classic TTS.

Conflicts of interest: none

Acknowledgements: Câmara Municipal de Loulé 


\section{References}

1. Nowak R, Fijalkowska M, Gilis-Malinowska N, et al.(2017) Left ventricular function after takotsubo is not fully recovered in longterm follow-up: A speckle tracking echocardiography study. Cardiol J.;24(1):57-64.

2. Cai LY, Addetia K, Medvedofsky D, Spencer KT. (2017) Myocardial strain may be useful in differentiating Takotsubo cardiomyopathy from left anterior descending coronary artery ischemia. Int J Cardiol;230:359-363.

3. Citro R, Rigo F, Ciampi Q, et al. (2011) Echocardiographic assessment of regional left ventricular wall motion abnormalities in patients with tako-tsubo cardiomyopathy: Comparison with anterior myocardial infarction. Eur J Echocardiogr;12(7):542549.

4. Heggemann F, Hamm K, Kaelsch T, et al. (2011) Global and regional myocardial function quantification in Takotsubo cardiomyopathy in comparison to acute anterior myocardial infarction using two- dimensional (2D) strain echocardiography. Echocardiography. 2011;28(7):715-719.

5. Meimoun P, Abouth S, Boulanger J, Luycx-Bore A, Martis S, Clerc J. (2014) Relationship between acute strain pattern and recovery in tako-tsubo cardiomyopathy and acute anterior myocardial infarction: a comparative study using twodimensional longitudinal strain. Int J Cardiovasc Imaging;30(8):1491-1500.

6. Caldeira D, Lopes LR, Carmona S, et al. (2015) Takotsubo cardiomyopathy, beyond ventriculography and classical bidimensional echocardiography. Int J Cardiol;182:381-383.

7. Marques N. (2019) Longitudinal strain by speckle tracking echocardiography: Is it useful in clinical practice for differentiating Takotsubo syndrome from anterior myocardial infarction? Rev Port Cardiol;38(4):267-268.

8. Pestana G, Tavares-Silva M, Sousa C, et al. (2019) Myocardial dysfunction in Takotsubo syndrome: More than meets the eye? Rev Port Cardiol.;38(4):261-266.

9. Citro R, Lyon AR, Meimoun P, et al. (2015) Standard and advanced echocardiography in takotsubo (stress) cardiomyopathy: Clinical and prognostic implications. J Am Soc Echocardiogr;28(1):57-74.

10. Sosa S, Banchs J. (2015) Early Recognition of Apical Ballooning Syndrome by Global Longitudinal Strain Using Speckle Tracking Imaging - The Evil Eye Pattern, a Case Series. Echocardiography. 2015;32(7):1184-1192.

11. Weiner MM, Asher DI, Augoustides JG, et al. (2017) Takotsubo Cardiomyopathy: A Clinical Update for the Cardiovascular Anesthesiologist. J Cardiothorac Vasc Anesth. 2017;31(1):334344.

12. Lyon AR, Bossone E, Schneider B, et al. (2016) Current state of knowledge on Takotsubo syndrome: A Position Statement from the Taskforce on Takotsubo Syndrome of the Heart Failure Association of the European Society of Cardiology. Eur J Heart Fail ;18(1):8-27.

13. Mansencal N, Abbou N, Pillière R, El Mahmoud R, Farcot JC, Dubourg O. (2009) Usefulness of Two- Dimensional Speckle Tracking Echocardiography for Assessment of Tako-Tsubo Cardiomyopathy. Am J Cardiol;103(7):1020-1024.
14. Kobayashi Y, Okura H, Kobayashi Y, Fukuda S, Hirohata A, Yoshida K. (2017) Left ventricular myocardial function assessedbythree-dimensionalspeckletracking echocardiography in Takotsubo cardiomyopathy. Echocardiography. 2017;34(4):523529.

15. Ghadri JR, Wittstein IS, Prasad A, et al. (2018) International Expert Consensus Document on Takotsubo Syndrome (Part I):

Clinical Characteristics, Diagnostic Criteria, and Pathophysiology. Eur Heart J. ;39(22):2032-2046.

16. Bellini G. Gomes C, Veras GJ. (2010) Two-Dimensional Strain in Takotsubo Cardiomyopathy. Arq Bras Cardiol:2009-2011.

17. Ishigaki D, Okuyama H, Yuki K, et al. (2012) Serial evaluation of left ventricular contraction and relaxation in Takotsubo cardiomyopathy by $2 \mathrm{D}$ speckle tracking echocardiography. J Med Ultrason. 2012;39(4):265-269.

18. Amzulescu MS, Langet H, Saloux E, et al. (2017) Head-to-Head Comparison of Global and Regional Two- Dimensional Speckle Tracking Strain Versus Cardiac Magnetic Resonance Tagging in a Multicenter Validation Study. Circ Cardiovasc Imaging;10(11):111.

19. Dias A, Franco E, Rubio M, et al. (2018) Usefulness of left ventricular strain analysis in patients with takotsubo syndrome during acute phase. Echocardiography. 2018;35(2):179-183.

20. Heggemann F, Weiss C, Hamm K, et al. (2009) Global and regional myocardial function quantification by two-dimensional strain in Takotsubo cardiomyopathy. Eur J Echocardiogr;10(6):760-764.

21. Meimoun P, Clerc J, Vincent C, et al. (2013) Non-invasive detection of tako-tsubo cardiomyopathy vs. acute anterior myocardial infarction by transthoracic Doppler echocardiography. Eur Heart J Cardiovasc Imaging;14(5):464-470.

22. Okura H. (2015) Echocardiographic assessment of takotsubo cardiomyopathy: beyond apical ballooning. J Echocardiogr. 2015;14(1):13-20.

23. Park S-M. (2009) Left Ventricular Systolic and Diastolic Function in Patients With Apical Balloning Syndrome Compared with Patients with Acute Anterior ST-Segment Elevation Myocardial Infarction : A Functional Paradox. Mayo Clin Proc;55905(June):514-521.

24. Heggemann F, Hamm K, Brade J, et al. (2014) Right ventricularfunctionquantificationinTakotsubo cardiomyopathy using two-dimensional strain echocardiography. PLoS One. 2014;9(8):1-8.

25. Vizzardi E, Bonadei I, Piovanelli B, Bugatti S, D’Aloia A. (2014) Biventricular Tako-Tsubo cardiomyopathy: Usefulness of 2D speckle tracking strain echocardiography. J Clin Ultrasound;42(2):121-124.

26. Uznañska B, Plewka M, Wierzbowska-Drabik K, Chrzanowski Ł, Kasprzak JD. (2009) Early prediction of ventricular recovery in takotsubo syndrome using stress and contrast echocardiography. Med Sci Monit;15(6):89-94.

27. Kim SA, Jo SH, Park KH, Kim HS, Han SJ, Park WJ. (2017) Functional recovery of regional myocardial deformation in patients with takotsubo cardiomyopathy. J Cardiol.;70(1):68-73. 